\title{
Deletion of the Correia element in the $m t r$ gene complex of Neisseria meningitidis
}

\begin{abstract}
Correspondence
Julio A. Vázquez

jvazquez@isciii.es
\end{abstract}

Received 8 April 2010

Accepted 8 June 2010
Rocío Enríquez, ${ }^{1}$ Raquel Abad, ${ }^{1}$ Grettel Chanto, ${ }^{2}$ Alejandra Corso, ${ }^{3}$ Raquel Cruces, ${ }^{1}$ Jean Marc Gabastou, ${ }^{4}$ María Cecilia Gorla, ${ }^{5}$ Aurora Maldonado, ${ }^{6}$ Jaime Moreno, ${ }^{7}$ Erwan Muros-Le Rouzic, ${ }^{8}$ Cecilia Sorhouet $^{3}$ and Julio A. Vázquez ${ }^{1}$

\author{
${ }^{1}$ Reference Laboratory for Meningococci, Centro Nacional de Microbiología, Instituto de Salud \\ Carlos III, Madrid, Spain \\ ${ }^{2}$ Centro Nacional de Referencia en Bacteriología (INCIENSA), San José, Costa Rica \\ ${ }^{3}$ Bacteriología, Instituto Nacional de Enfermedades Infecciosas (INEI-ANLIS) Dr C. G. Malbrán, \\ Buenos Aires, Argentina \\ ${ }^{4}$ Unidad de Medicamentos Esenciales, Vacunas y Tecnologías de Salud, Pan American Health \\ Organization (PAHO)/World Health Organization (WHO), Quito, Ecuador \\ ${ }^{5}$ Bacteriology Branch, Adolfo Lutz Institute, São Paulo, Brazil \\ ${ }^{6}$ Bacteriología, Instituto de Salud Pública (ISP), Santiago de Chile, Chile \\ ${ }^{7}$ Microbiología, Instituto Nacional de Salud (INS), Bogotá, Colombia \\ ${ }^{8}$ Global Scientific \& Medical Affairs, Sanofi-Pasteur, Lyon, France
}

The $m t r$ gene complex in Neisseria meningitidis encodes an efflux pump that is responsible for export of antibacterial hydrophobic agents. The promoter region of the $m t r C D E$ operon harbours an insertion sequence known as a Correia element, and a binding site for the integration host factor (IHF) is present at the centre of the Correia element. It has been suggested that the expression of the mtrCDE operon in meningococci is subject to transcriptional regulation by the IHF and post-transcriptional regulation by cleavage in the inverted repeat of the Correia element. The promoter region of the $m \operatorname{tr} C D E$ operon as well as the association of changes at that point with decreased susceptibility to antimicrobial drugs in 606 Neisseria meningitidis strains were analysed in this study. Two different deletions were present in the analysed region. The first one, found in seven strains, corresponded to absence of the Correia element. The second one, affecting the -10 region and first $100 \mathrm{bp}$ of the $m t r R$ gene and present in 57 isolates, was only found in ST-1624 isolates. None of the deletions were associated with decreased susceptibility to antimicrobial drugs. Although most of the meningococcal strains carry the Correia element at that position, its deletion is not an exception.

\section{INTRODUCTION}

The $m t r$ gene complex encodes an energy-dependent efflux pump that is responsible for export of hydrophobic agents, including those with a non-ionic detergent-like activity, antibiotics and antibacterial peptides. This efflux pump is composed of the MtrC, MtrD and MtrE proteins, which are encoded by the tandemly linked mtrCDE genes. In Neisseria gonorrhoeae, the mtrCDE operon is negatively regulated by the product of the adjacent but divergent $m t r R$ gene positioned $250 \mathrm{bp}$ upstream. Mutations in the

Abbreviations: CC, clonal complex; IHF, integration host factor; MLST, multilocus sequence typing; ORDR, quinolone-resistance determining region; ST, sequence type. $m t r R$ gene or in its promoter enhance $m \operatorname{tr} C D E$ gene expression, leading to gonococcal clinical isolates that express elevated levels of resistance to hydrophobic agents (Dewi et al., 2004; Rouquette-Loughlin et al., 2004).

The emergence of meningococcal strains with reduced susceptibility to ciprofloxacin has only been reported occasionally (Enríquez et al., 2008). This reduced susceptibility has been associated with point mutations in the quinolone-resistance determining regions (QRDRs) of the target sites for the fluoroquinolones, and particularly with changes in the GyrA subunit of DNA gyrase (Shultz et al., 2005). However, point mutations at that locus or in the other QRDRs (parC, gyrB and parE) were not detected in two meningococcal strains isolated in Argentina and Spain 
showing an MIC for ciprofloxacin of 0.12 and $0.25 \mathrm{mg} \mathrm{l}^{-1}$, respectively. Both strains [characterized as serogroup Y and assigned to sequence type (ST)-1624 from the ST-167 clonal complex (CC)] showed a 154 bp deletion affecting the $m t r R$ gene from the mtrRCDE gene complex. This deletion was suggested as responsible for reduced susceptibility to ciprofloxacin in these strains (Enríquez et al., 2008; Corso et al., 2005).

Two independent genome sequencing projects have verified the presence of the Mtr efflux system in Neisseria meningitidis (Parkhill et al., 2000; Tettelin et al., 2000) and recently Rouquette-Loughlin et al. (2004) reported that meningococci have a functional MtrCDE efflux system but its expression is independent of MtrR. The authors analysed the promoter region of the $m t r C D E$ operon in several meningococcal strains and found it contained a $155 \mathrm{bp}$ and $159 \mathrm{bp}$ insertion sequence that corresponded to the previously described Correia element placed immediately downstream of the $m t r C D E$ promoter (Rouquette-Loughlin et al., 2004). Correia elements are small insertion sequences (100-155 bp in length) of unknown function that have long-terminal inverted repeats and a target site duplication, and have been identified elsewhere in the gonococcal and meningococcal genomes (Delahay et al., 1997; Buisine et al., 2002). Buisine et al. (2002) reported the presence of an integration host factor (IHF) binding site at the centre of the Correia element (Buisine et al., 2002). The IHF was found to bind specifically to this site and deletion of the IHF binding site within the Correia element enhanced mtrCDE transcription. Post-transcriptional regulation of the $m t r C D E$ transcript by cleavage in the inverted repeat of the Correia element has also been reported.

In this study, the presence of deletions in $m t r R$ gene, their frequency in the meningococcal population and their potential association with the decreased susceptibility to antimicrobial drugs were analysed in 606 meningococcal strains from different CCs.

\section{METHODS}

N. meningitidis strains. The analysis included 606 meningococcal strains associated with patients and also carriers, isolated in six countries (Spain, Argentina, Brazil, Chile, Colombia and Costa Rica) from 1992 to 2007. The strains belonged to different serogroups and represented different CCs (Table 1).

PCR amplification and DNA sequencing. Previously recommended primers mtr405 and mtr254 (Stefanelli et al., 2001) were used to amplify a $1033 \mathrm{bp}$ fragment corresponding to the first $27 \mathrm{bp}$ of the $m t r C$ gene, the $m t r R / m t r C D E$ intergenic region containing the Correia element and the putative promoters of the $m t r R$ and $m t r C D E$ genes, and the first $800 \mathrm{bp}$ of the $m t r R$ gene (Fig. 1a). The PCR products were analysed by agarose gel electrophoresis for checking the presence of deletions by comparison of the sizes of the amplified fragments (Fig. 1b). Strains harbouring deletions were sequenced using the same pair of primers. Fifty-five strains without deletions were also sequenced for comparison.

Alignments were made using the MEGALIGN program (DNASTAR).

Antimicrobial susceptibility testing. MICs of erythromycin, ciprofloxacin, rifampicin, penicillin, ceftriaxone and cefotaxime for 418 strains which are part of the Spanish collection were determined by the agar dilution method as has been previously described (Enríquez et al., 2008). Streptococcus pneumoniae ATCC 49619 and Escherichia coli ATCC 25922 were used as quality control organisms.

MICs of erythromycin, ciprofloxacin and rifampicin for a selection of 20 meningococcal strains isolated in Latin America (including strains with and without deletions in the amplified region) were determined by the Etest method ( $\mathrm{AB}$ Biodisk) according to the manufacturer's recommendations.

The breakpoints used were those recommended by the CLSI for Neisseria meningitidis (CLSI, 2007).

Table 1. Meningococcal strains included in the screening for the $m t r$ deletion

\begin{tabular}{|lcccc|}
\hline Serogroup & Country of isolation & No. of strains & mtrR deletion & ST/CC $\dagger$ \\
\hline A & Spain & 7 & 0 & $4031 / 41 / 44$ Lineage 3 \\
B & Spain & 108 & $1(152 \mathrm{bp})$ & $35 /$ ST-35 \\
C & Spain & 96 & $1(152 \mathrm{bp})$ & $23 /$ ST-23 \\
$29 \mathrm{E}$ & Spain & 23 & 0 & $1624 /$ ST-167 \\
Y & Spain & 78 & $3 \ddagger(152 \mathrm{bp})$ & $6533 /$ ST-23 \\
Y & Argentina & 63 & $52(154 \mathrm{bp})$ & $1624 /$ ST-167 \\
Y & Brazil & 44 & $2(152 \mathrm{bp})$ & \\
Y & Chile & 23 & 0 & \\
Y & Colombia & 41 & 0 & 0 \\
Y & Costa Rica & 16 & 0 & \\
W135 & Spain & 96 & 11 & \\
X & Spain & & & \\
\end{tabular}

${ }^{\star}$ No. of strains with $m t r R$ deletion and type of deletion.

$\dagger$ ST corresponds to the assigned sequence type; CC corresponds to the clonal complex in which the ST has been included.

$\ddagger$ Two strains were isolated from asymptomatic carriers. 


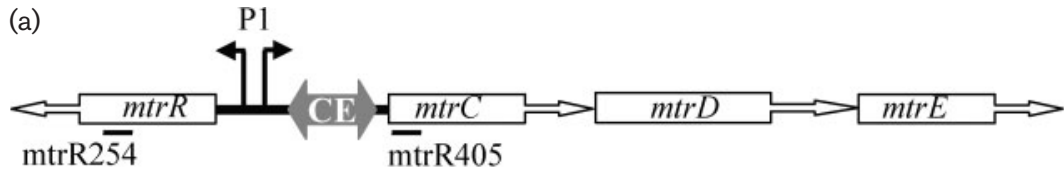

154 bp deletion ... 152 bp deletion

(b)

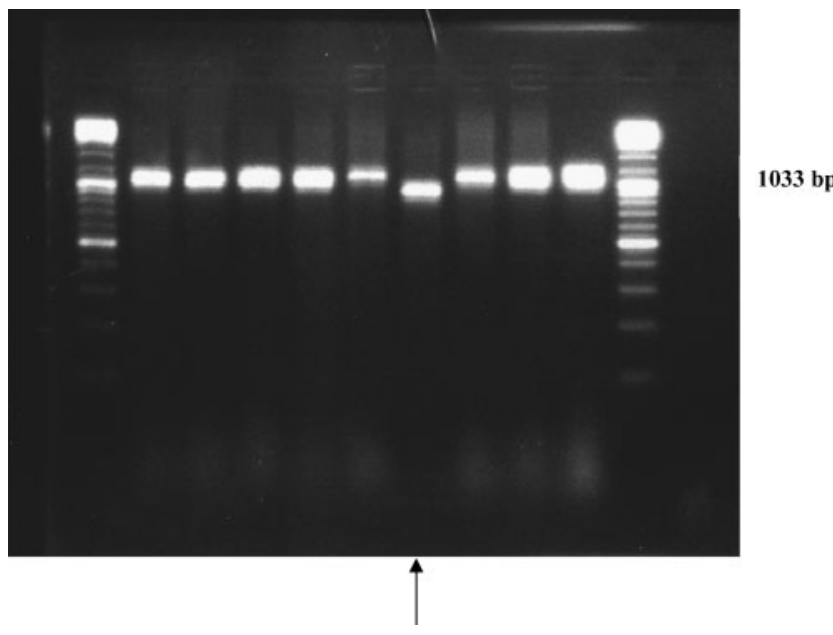

Fig. 1. (a) Schematic organization of the $m t r$ locus in $N$. meningitidis. The solid arrows represent the putative promoters of the $m t r R$ and $m t r C D E$ genes $(\mathrm{P} 1)$. CE, Correia element. The location of the primers used is shown with a solid line. The location of the two different deletions is also indicated. (b) Agarose gel showing the wild-type fragment and one strain with a deletion marked with an arrow.

\begin{abstract}
Multilocus sequence typing (MLST). MLST was performed in 372 strains (all strains showing deletions and 308 randomly chosen strains) as described by Maiden et al. (1998). The ST and the CC designation was done according to the Neisseria MLST website (http://pubmlst.org/neisseria/). All STs already assigned to the ST-167 CC (to which ST-1624 belongs) on the Allelic Profile/ST Database hosted in http://pubmlst.org/neisseria were used to build an eBURST representation for that CC (Fig. 2).
\end{abstract}

\section{RESULTS AND DISCUSSION}

\section{Mutations in the mtrCDE gene complex}

The panel of meningococci included in the study corresponds to a quite diverse meningococcal population with strains showing seven serogroups (Table 1) and belonging to more than 164 STs (data not shown).

After analysis of the amplified region (Fig. 1b), deletions were found in 64 of the $606 \mathrm{~N}$. meningitidis strains studied. The amplified region was sequenced in all strains (64) harbouring deletions and also in 55 strains without deletion for comparison, and two different deletions were identified (Fig. 1a). The first one, a 152 bp deletion found in seven strains, corresponded to absence of the Correia element on the intergenic region (GenBank accession no. GQ424833). The second one, a 154 bp deletion found in 57 strains, affected the -10 region and the first $100 \mathrm{bp}$ of the $m$ trR gene (GenBank accession no. EF117896).

The $152 \mathrm{bp}$ deletion, corresponding to absence of the Correia element, was found in seven strains showing different serogroups and belonging to different STs (Table 1): five strains were serogroup Y, three belonging to ST-23 (CC ST-23/Cluster A3) and two belonging to ST-6533 (CC ST-23/Cluster A3); one strain was serogroup B, ST-4031 (CC ST-41/44 Lineage 3); and the other strain was serogroup C, ST-35 (CC ST-35). Two of these seven strains were isolated from carriers so it is likely that this event can not be associated with strain virulence.

Several authors have identified the Correia element in the $m t r R / m t r C D E$ intergenic region in meningococcal strains (Rouquette-Loughlin et al., 2004; Parkhill et al., 2000; Tettelin et al., 2000; Abadi et al., 1996), suggesting that this insertion sequence might be a common trait in meningococcal isolates, and Rouquette-Loughlin et al. (2004) have proposed that it could play a role in modulation of $m t r C D E$ expression. The results presented here indicate that the Correia element is not present at this location in all meningococcal strains. Although most of the meningococcal strains may carry the Correia element at that position, the absence of this element is not an exception. The $154 \mathrm{bp}$ deletion, affecting the -10 region and first $100 \mathrm{bp}$ of the mtrR gene, was only found in serogroup Y strains belonging to ST-1624 (CC ST-167) (Table 1), all of them being isolated from patients. All the strains characterized as Y and ST-1624 showed this deletion. To find out whether the $154 \mathrm{bp}$ deletion could be used as a ST-1624 or CC ST-167 marker, the deletion in 15 strains (belonging to the collection of 606 strains included in this study) belonging to 15 different STs from CC ST-167 (Fig. 2) was checked. None of the analysed strains 


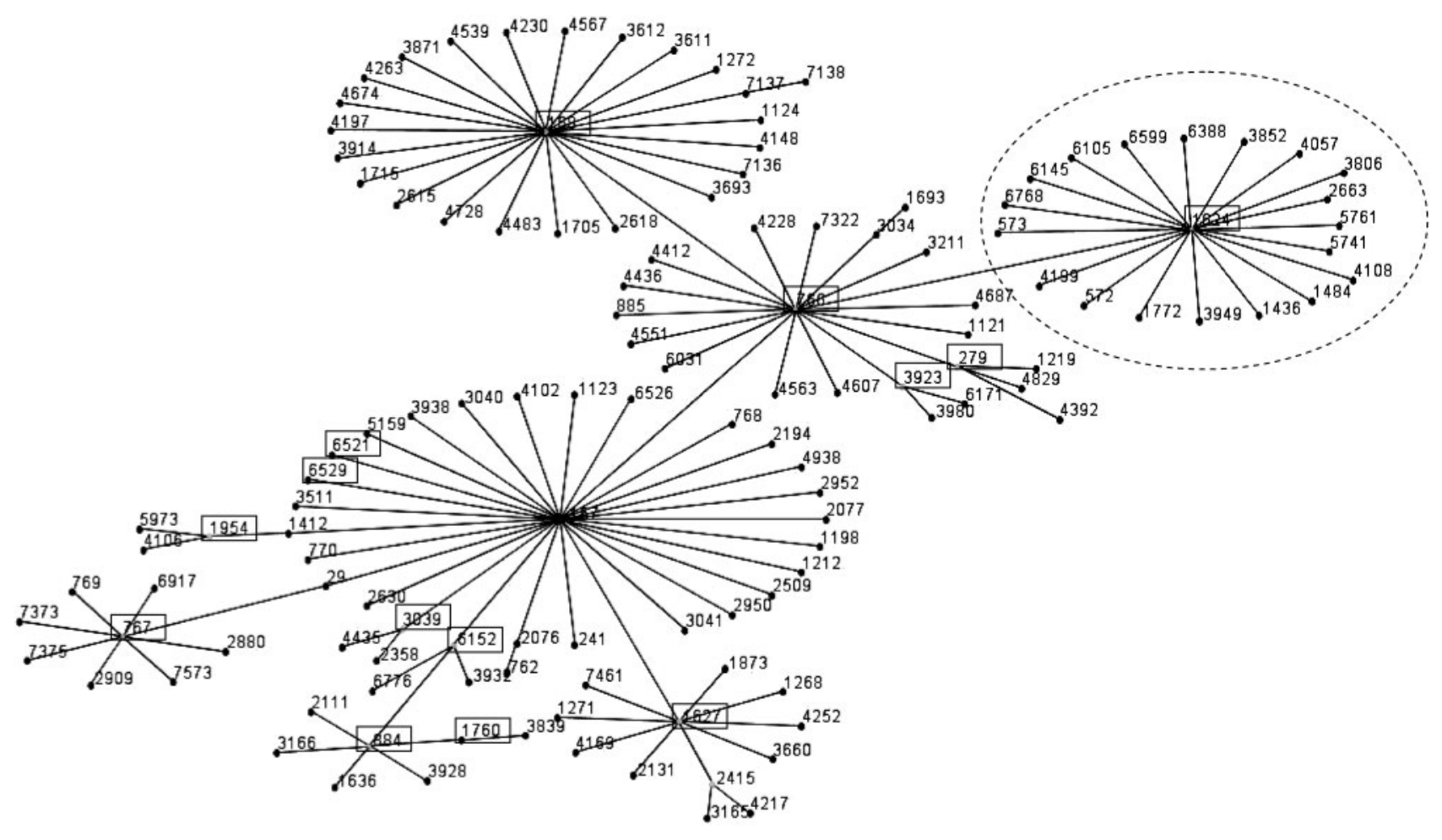

Fig. 2. eBURST representation of the ST-167 CC. Those STs in which at least one strain was analysed appear framed with a solid line. ST-167 and STs potentially evolving from ST-167 appear framed with a broken line.

belonging to the potential ancestors for ST-1624 presented the deletion. We do not have strains of the STs evolving from ST-1624, so we can not say whether the deletion is present in all STs potentially descending from ST-1624. Therefore, we can conclude that this particular deletion might have arisen in ST-1624 and it is a special feature of this ST.

\section{Susceptibility to antimicrobial agents}

There were no differences when the MICs of erythromycin, ciprofloxacin or rifampicin among strains both with and without deletions were compared (Table 2). All the analysed strains were susceptible to these antibiotics. Therefore, neither of the two deletions found can be associated with decreased susceptibility to antimicrobial agents.

A full-length MtrR protein is needed for regulation of $m t r C D E$ in gonococci (Rouquette-Loughlin et al., 2004). While the MtrR protein acts as a negative transcriptional regulator in $N$. gonorrhoeae and mutations in the $m t r R$ gene or in its promoter lead to resistance to hydrophobic agents (Dewi et al., 2004; Rouquette-Loughlin et al., 2004), the deletion of $154 \mathrm{bp}$ affecting the -10 region and first $100 \mathrm{bp}$ of the $m t r R$ gene observed was not associated with reduced susceptibility to antimicrobial agents. RouquetteLoughlin et al. (2004) analysed the promoter region of the $m t r C D E$ operon in a panel of meningococcal strains and they reported the presence of a 155-159 bp insertion sequence element, known as the Correia element, placed immediately downstream of the $m t r C$ promoter in all meningococcal strains tested. However, it seems to be a rare phenomenon in gonococci. The authors concluded that the presence of a Correia element within the mtrR/ $m t r C$ intergenic region is a common trait in meningococcal isolates. However, the $152 \mathrm{bp}$ deletion found in seven of the strains analysed in this study revealed that the Correia element is not always present at this location in the meningococcal population.

Post-transcriptional regulation of the $m \operatorname{tr} C D E$ transcript by cleavage in the inverted repeat of the Correia element has been proposed (Rouquette-Loughlin et al., 2004). The $152 \mathrm{bp}$ deletion, corresponding to absence of the Correia element, also involved the IHF binding site at the centre of the Correia element that has been suggested as critical for modulating expression of MtrCDE. Those seven strains harbouring the deletion did not show decreased susceptibility to the antimicrobial agents tested. We have not checked the level of expression of MtrCDE in those meningococci showing absence of the Correia element at that position. The lack of an MIC increase does not necessarily prove that the $m t r$ efflux system in meningococci is not subject to transcriptional regulation by IHF. The deletions described might have resulted in changes in the expression of the MtrCDE system without translation into a significant MIC change in meningococci. Additional 
Table 2. MICs of erythromycin, ciprofloxacin and rifampicin in a selection of meningococcal strains with and without deletions in the $m$ tr gene complex

\begin{tabular}{|c|c|c|c|c|}
\hline \multirow[t]{2}{*}{ ID number } & \multirow[t]{2}{*}{ Deletion } & \multicolumn{3}{|c|}{$\operatorname{MICs}\left(\mathrm{mg} \mathrm{l}^{-1}\right)$} \\
\hline & & Erythromycin & Ciprofloxacin & Rifampicin \\
\hline AR930 & $154 \mathrm{bp}$ & 0.25 & 0.006 & 0.003 \\
\hline 1055 & - & 1 & 0.006 & 0.012 \\
\hline 1730 & $154 \mathrm{bp}$ & 0.5 & 0.006 & 0.006 \\
\hline 1964 & $154 \mathrm{bp}$ & 0.25 & 0.006 & 0.003 \\
\hline 2096 & $154 \mathrm{bp}$ & 0.25 & 0.006 & 0.006 \\
\hline 2151 & $154 \mathrm{bp}$ & 0.25 & 0.006 & 0.003 \\
\hline 2289 & $154 \mathrm{bp}$ & 0.5 & 0.006 & 0.003 \\
\hline 2432 & $154 \mathrm{bp}$ & 0.25 & 0.006 & 0.006 \\
\hline 2508 & $154 \mathrm{bp}$ & 0.5 & 0.006 & 0.006 \\
\hline $\mathrm{N} 21 / 05$ & - & 0.5 & 0.006 & 0.003 \\
\hline $\mathrm{N} 71 / 00$ & - & 0.5 & 0.006 & 0.006 \\
\hline N207/05 & - & 0.5 & 0.006 & 0.003 \\
\hline N683/05 & $152 \mathrm{bp}$ & 0.5 & 0.006 & 0.012 \\
\hline N689/05 & $152 \mathrm{bp}$ & 0.5 & 0.006 & 0.012 \\
\hline 13977 & $152 \mathrm{bp}$ & 0.25 & 0.006 & 0.006 \\
\hline 14203 & $152 \mathrm{bp}$ & 0.5 & 0.006 & 0.012 \\
\hline 15103 & - & 0.25 & 0.006 & 0.025 \\
\hline 15320 & - & 0.25 & 0.006 & 0.006 \\
\hline 15596 & - & 0.5 & 0.006 & 0.012 \\
\hline 15656 & $152 \mathrm{bp}$ & 1 & 0.006 & 0.012 \\
\hline 15701 & - & 0.5 & 0.006 & 0.012 \\
\hline 15970 & $152 \mathrm{bp}$ & 1 & 0.006 & 0.025 \\
\hline $422 \mathrm{~N}$ & - & 0.5 & 0.006 & 0.012 \\
\hline $513 \mathrm{~N}$ & - & 0.25 & 0.006 & 0.006 \\
\hline $\mathrm{M} 12 / 02$ & $154 \mathrm{bp}$ & 0.25 & 0.003 & 0.012 \\
\hline M25/01 & $154 \mathrm{bp}$ & 0.25 & 0.003 & 0.012 \\
\hline M102/04 & $154 \mathrm{bp}$ & 0.25 & 0.003 & 0.012 \\
\hline
\end{tabular}

studies on the expression of the efflux system in those meningococci are necessary to elucidate the exact meaning of the deletions found. However, the hypothetical association of the 154 bp deletion with quinolone resistance in strains without changes in QRDRs (Corso et al., 2005; Enríquez et al., 2008) is rejected by this study. Alternative mechanisms for quinolone resistance in those meningococcal isolates should be considered and further analysed.

\section{ACKNOWLEDGEMENTS}

This work made use of the MLST website (www.mlst.net). This study was partially supported in different aspects by the Ministerio de Ciencia e Innovación, ISCIII, Spanish Network for the Research in Infectious Diseases (REIPI RD06/0008), by Sanofi-Pasteur SA, through an institutional agreement with the Institute of Health Carlos III, and by the Spanish Fund for Health Research (FIS PI1061346)

\section{REFERENCES}

Abadi, F. J., Carter, P. E., Cash, P. \& Pennington, T. H. (1996). Rifampin resistance in Neisseria meningitidis due to alterations in membrane permeability. Antimicrob Agents Chemother 40, 646-651.
Buisine, N., Tang, C. M. \& Chalmers, R. (2002). Transposon-like Correia elements: structure, distribution and genetic exchange between pathogenic Neisseria sp. FEBS Lett 522, 52-58.

CLSI (2007). Performance Standards for Antimicrobial Susceptibility Testing, 15th Informational Supplement, M100-S15. Wayne, PA: Clinical and Laboratory Standards Institute.

Corso, A., Faccone, D., Miranda, M., Rodriguez, M., Regueira, M., Carranza, C., Vencina, C., Vazquez, J. A. \& Galas, M. (2005). Emergence of Neisseria meningitidis with decreased susceptibility to ciprofloxacin in Argentina. J Antimicrob Chemother 55, 596-597.

Delahay, R. M., Robertson, B. D., Balthazar, J. T., Shafer, W. M. \& Ison, C. A. (1997). Involvement of the gonococcal MtrE protein in the resistance of Neisseria gonorrhoeae to toxic hydrophobic agents. Microbiology 143, 2127-2133.

Dewi, B. E., Akira, S., Hayashi, H. \& Ba-Thein, W. (2004). High occurrence of simultaneous mutations in target enzyme and MtrRCDE efflux system in quinolone-resistant Neisseria gonorrhoeae. Sex Transm Dis 31, 353-359.

Enríquez, R., Abad, R., Salcedo, C., Pérez, S. \& Vázquez, J. A. (2008). Fluoroquinolone resistance in Neisseria meningitidis in Spain. J Antimicrob Chemother 61, 286-290.

Maiden, M. C., Bygraves, J. A., Feil, E., Morelli, G., Russell, J. E., Urwin, R., Zhang, O., Zhou, J., Zurth, K. \& other authors (1998). Multilocus sequence typing: a portable approach to the identification of clones within populations of pathogenic microorganisms. Proc Natl Acad Sci U S A 95, 3140-3145. 
Parkhill, J., Achtman, M., James, K. D., Bentley, S. D., Churcher, C., Klee, S. R., Morelli, G., Basham, D., Brown, D. \& other authors (2000). Complete DNA sequence of a serogroup A strain of Neisseria meningitidis Z2491. Nature 404, 502-506.

Rouquette-Loughlin, C. E., Balthazar, J. T., Hill, S. A. \& Shafer, W. M. (2004). Modulation of the mtrCDE-encoded efflux pump gene complex of Neisseria meningitidis due to a Correia element insertion sequence. Mol Microbiol 54, 731-741.

Shultz, T. R., White, P. A. \& Tapsall, J. W. (2005). In vitro assessment of the further potential for development of fluoroquinolone resistance in Neisseria meningitidis. Antimicrob Agents Chemother 49, 17531760.

Stefanelli, P., Fazio, C., La Rosa, G., Marianelli, C., Muscillo, M. \& Mastrantonio, P. (2001). Rifampicin-resistant meningococci causing invasive disease: detection of point mutations in the rpoBgene and molecular characterization of the strains. J Antimicrob Chemother 47, 219-222.

Tettelin, H., Saunders, N. J., Heidelberg, J., Jeffries, A. C., Nelson, K. E., Eisen, J. A., Ketchum, K. A., Hood, D. W., Peden, J. F. \& other authors (2000). Complete genome sequence of Neisseria meningitidis serogroup B strain MC58. Science 287, 1809-1815. 